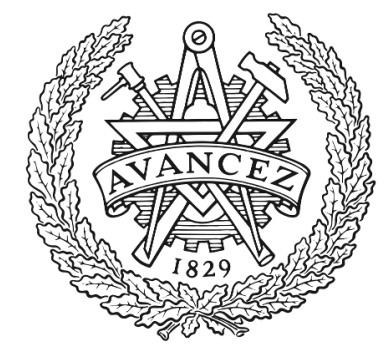

CHALMERS

UNIVERSITY OF TECHNOLOGY

\title{
Computation of the Exact Bit-Error Rate of Coherent M-ary PSK With Gray Code Bit Mapping
}

Downloaded from: https://research.chalmers.se, 2023-04-26 10:44 UTC

Citation for the original published paper (version of record):

Lassing, J., Ström, E., Agrell, E. et al (2003). Computation of the Exact Bit-Error Rate of Coherent M-ary PSK With Gray Code Bit Mapping. IEEE Transactions on Communications, 51(11):

1758-1760. http://dx.doi.org/10.1109/TCOMM.2003.818102

N.B. When citing this work, cite the original published paper.

C2003 IEEE. Personal use of this material is permitted.

However, permission to reprint/republish this material for advertising or promotional purposes 


\section{Computation of the Exact Bit Error Rate of Coherent $M$-ary PSK with Gray Code Bit Mapping}

Johan Lassing, Erik G. Ström, Erik Agrell and Tony Ottosson 


\begin{abstract}
The problem of calculating the average bit-error probability (BER) of coherent $M$-ary phase shift keying (PSK) over a Gaussian channel has been studied previously in the literature. A solution to the problem for systems using a binary reflected Gray code (BRGC) to map bits to symbols was first presented by Lee in [1]. In this letter we show that the results obtained in [1] are incorrect for $M \geq 16$. We show that the reason for this is an invalid assumption that the BER is independent of the transmitted symbols, an assumption which has also propagated to textbooks, e.g., [2, p. 211]. We give a new expression for the BER of $M$-PSK systems using the BRGC and compare this with the results of [1].
\end{abstract}

\title{
I. INTRODUCTION
}

In this letter we will revise the calculation of the bit error probability of $M$-PSK, as presented in [1]. We adopt the same vector model as in [1] and we show how to put the results from [1] into a convenient form and then give a new expression for the BER of a binary reflected Gray encoded $M$-PSK system as a function of $M$.

\section{AN EXPRESSION FOR THE AVERAGE BER OF $M$-PSK}

For any mapping between bits and symbols, the BER is given by the expression

$$
P_{\mathrm{b}}=\sum_{l=0}^{M-1} \sum_{\substack{k=0 \\ k \neq l}}^{M-1} \frac{d\left(\mathbf{c}_{k}, \mathbf{c}_{l}\right)}{m} \operatorname{Pr}\left\{\mathbf{r} \in S_{k} \mid \mathbf{s}=\mathbf{s}_{l}\right\} \operatorname{Pr}\left\{\mathbf{s}=\mathbf{s}_{l}\right\}
$$

Here $\mathbf{s}_{k}, k=0,1, \ldots, M-1$, denote the $M=2^{m}$ available signal vectors (symbols) and $S_{k}$ is the decision region for symbol $\mathbf{s}_{k}$. The binary vector $\mathbf{c}_{k}, k=0,1, \ldots, M-1$, denotes the bit pattern assigned to the symbol $\mathbf{s}_{k}$, while the function $d\left(\mathbf{c}_{k}, \mathbf{c}_{l}\right)$ denotes the Hamming distance between the binary vectors $\mathbf{c}_{k}$ and $\mathbf{c}_{l}$, i.e., the number of positions in which they differ. The number of bits in the bit vectors $\mathbf{c}_{k}$ is $m=\log _{2} M$.

We assume (as in [1]) that the $M$ symbols are spread uniformly on a circle of radius $\sqrt{E_{s}}=\sqrt{m E_{b}}$ in the signal space, where $E_{s}$ and $E_{b}$ are the symbol and bit energy, respectively. Furthermore, we assume (also as in [1]) that the symbols are equally likely to be transmitted, and that the receiver is optimum in the sense that symbol error probability (SER) is minimized. This implies that all decision regions will be geometrically congruent and that the probabilities $\operatorname{Pr}\left\{\mathbf{r} \notin S_{k} \mid \mathbf{s}=\mathbf{s}_{k}\right\}$ will be equal, independent of $k$. Using these assumptions, we can rewrite (1) as

$$
\begin{aligned}
& P_{\mathrm{b}}=\sum_{l=0}^{M-1} \sum_{k=1}^{M-1} \frac{d\left(\mathbf{c}_{l}, \mathbf{c}_{(l+k) \bmod M)}\right.}{m} \underbrace{\operatorname{Pr}\left\{\mathbf{r} \in S_{(l+k) \bmod M} \mid \mathbf{s}=\mathbf{s}_{l}\right\}}_{\triangleq P(k)} \underbrace{\operatorname{Pr}\left\{\mathbf{s}=\mathbf{s}_{l}\right\}}_{=1 / M} \\
& =\frac{1}{m} \sum_{k=1}^{M-1} P(k) \underbrace{\sum_{l=0}^{M-1} \frac{d\left(\mathbf{c}_{l}, \mathbf{c}_{(l+k) \bmod M)}\right.}{M}}_{\triangleq \bar{d}(k)}=\frac{1}{m} \sum_{k=1}^{M-1} \bar{d}(k) P(k)
\end{aligned}
$$


Here we have introduced the average distance spectrum $\bar{d}(k)$ to denote the average number of bit positions differing between signal alternatives separated by $k$ steps along the circle, i.e.,

$$
\begin{aligned}
\bar{d}(k) & =\frac{1}{M} \sum_{l=0}^{M-1} d\left(\mathbf{c}_{l}, \mathbf{c}_{(l+k) \bmod M}\right) \\
& =\frac{1}{M} \sum_{l=0}^{M-1} \sum_{i=0}^{m-1}\left|\left[\mathbf{c}_{l}\right]_{i}-\left[\mathbf{c}_{(l+k) \bmod M}\right]_{i}\right|
\end{aligned}
$$

where the notation $\left[\mathbf{c}_{l}\right]_{i}$ is used to denote the $i$ th element of the binary vector $\mathbf{c}_{l}$. Obviously, it is the average distance spectrum $\bar{d}(k)$ that depends on the particular bit-to-symbol mapping chosen, and traditionally this is evaluated using computer simulation [3]. In addition, textbooks often use approximations to obtain expressions for the BER as a function of $M$, see [4, p. 202] [5]. The probabilities $P(k)$ can be evaluated using the expressions given in, e.g., [4, p. 201] [1,6].

By comparing the final expression in (2) with (5) in [1], we see that the equations are similar. However, the important difference is that [1] uses simply the Hamming weight of the binary vector $\mathbf{c}_{k}$ (i.e., the Hamming distance between $\mathbf{c}_{k}$ and the all-zero codeword $\mathbf{c}_{0}$ ) to multiply the corresponding $P(k)$, whereas in our (2), it is the average Hamming distance difference taken over all binary vectors separated by $k$ steps that multiplies each $P(k)$. The motivation for using the weight directly in [1] is the claim that the BER is independent of the transmitted symbols. If this claim were correct, then the Hamming weight spectrum would be identical to the average distance spectrum. We show below that it is not.

\section{Evaluating the Average Distance Spectrum}

In this section we will present a closed-form expression for the average distance spectrum $\bar{d}(k)$ for the binary reflected Gray code (BRGC), which is the particular bit-to-symbol mapping used in [1].

The BRGC is what is called a cyclic Gray code, meaning that in the list containing the codewords all adjacent codewords differ in exactly one bit position, including the first and the last codeword in the list. This property is desirable for $M$-PSK, where we want to label the quantization of a circle. The BRGC scheme, originally proposed and patented by Frank Gray in 1953 [7], is actually only one special code in a large class of codes having the property that adjacent codewords differ in only one position [8,9]. For $m=1(M=2)$, the BRGC is simply $\{0,1\}$. The BRGC of order $m$ can be constructed recursively from the BRGC of order $m-1$ according to the following procedure: $(i)$ list the $M / 2=2^{m-1}$ codewords of the BRGC of order $m-1$ two times row-wise over each other, first in the original order and then in reflected (reverse) order, then ( $i i)$ add a column to the left of the list consisting of $M / 2$ zeros followed by $M / 2$ ones. This is illustrated for the BRGCs of order $m=1,2,3$, and 4 in Table I. 
To obtain the average distance spectrum $\bar{d}(k)$, we first exchange the order of summation in (3) to get

$$
\bar{d}(k)=\sum_{i=0}^{m-1}\left(\frac{1}{M} \sum_{l=0}^{M-1}\left|\left[\mathbf{c}_{l}\right]_{i}-\left[\mathbf{c}_{(l+k) \bmod M}\right]_{i}\right|\right)=\sum_{i=0}^{m-1} \bar{d}_{i}(k)
$$

where $\bar{d}_{i}(k)$ is the average number of bits that change in position $i$ of bit patterns separated by $k$ steps along the circle.

As is shown in Appendix, the recursive construction method leads to an expression for the average distance spectrum of the BRGC of order $m$ given by

$$
\bar{d}(k)=\sum_{i=0}^{m-1} \bar{d}_{i}(k)=\operatorname{tri}\left(2^{m}, k\right)+\sum_{j=2}^{m} \operatorname{tri}\left(2^{j}, k\right)
$$

which is valid for all integers $k$. The function $\operatorname{tri}(P, k)$ is a periodic triangular function of period $P$, given by

$$
\operatorname{tri}(P, k)=2\left|\frac{k}{P}-\left\lfloor\frac{k}{P}\right\rceil\right|
$$

where the function $\lfloor x\rceil$ rounds $x$ to the closest integer. If $x$ falls exactly midway between adjacent integers either of the two can be selected - the choice does not affect the value of $\operatorname{tri}(P, k)$. Inserting (6) into (5) we obtain

$$
\bar{d}(k)=2\left|\frac{k}{M}-\left\lfloor\frac{k}{M}\right\rceil\right|+2 \sum_{i=2}^{m}\left|\frac{k}{2^{i}}-\left\lfloor\frac{k}{2^{i}}\right\rceil\right|
$$

In Table II, $\bar{d}(k)$ is listed for $M=2,4,8$, and 16. The most interesting part of this table is the values for $M=16$, especially the non-integer values $\bar{d}(5)=\bar{d}(7)=2.5$ marked by asterisks. These values indicate that when the bit-to-symbol mapping is done using a BRGC, the bit error probability of $M$-PSK is not independent of the transmitted symbols. Obviously, if it would be, then only integer numbers could occur in the sequence $\bar{d}(k)$.

\section{RESUlts AND COMMENTS}

This section gives a plot of the average BER of binary reflected Gray coded $M$-PSK as a function of the bit-energy-to-noise ratio. The expression we evaluate is given by (2), where the average distance spectrum $\bar{d}(k)$ is given by (7) and the probabilities $P(k)$ are given in [1] (in which they are called $A_{k}$ ). Note that [1] does not explicitly list the sequences given in Table II, but inserting the sequences given in the table, using the relation $\bar{d}(k)=\bar{d}(M-k)$, into (5) in [1] will reproduce the results of (7)-(9) in [1].

In Figure $1, P_{\mathrm{b}}$ is evaluated for $M=16$ and for $\bar{d}(k)$ we use the values given for $M=16$ in Table II. From Figure 1 we see that the result presented in [1] gives a slight overestimation of the 
true BER. For reference, we include computer simulated values (o), which are seen to be in very good agreement with the new results derived in this paper. However, we also note that the difference between the previous result and the result derived here is small and vanishes with increasing $E_{b} / N_{0}$, since the two distance spectra first differ in the fifth position. This is probably the reason why the error in [1] has not been noted before. The discrepancy is a result of the invalid assumption that for the BRGC the BER is independent of the transmitted symbols, which is only valid for $1 \leq m \leq 3$. The results presented in [1] and also in [2, p. 211] and [4, p. 201] are incorrect for all $M \geq 16$ and instead (2) should be used together with (7) to obtain correct results (for any $M$ ). In [3] an alternative approach to deriving expressions for the BER results in the same (numerical) values as the expressions given here, but no general form for the average distance spectrum is provided.

\section{CONCLusions}

The main contribution of this paper is a closed-form expression for the average distance spectrum as a function of $M$ for the binary reflected Gray mapping given by (7). Since the derived expression differs from the results previously proposed in [1], we also correct these results here. The difference between the previous results and the new results derived herein is small, and we show that the erroneous results resulted from an invalid assumption that the BER is independent of the transmitted symbols.

\section{APPENDIX}

\section{DERIVATION OF AVERAGE DISTANCE SPECTRUM FOR BRGCS}

For any binary periodic sequence $b_{l}$ with period $P$ we have

$$
\bar{d}(b, k) \triangleq \frac{1}{P} \sum_{l=0}^{P-1}\left|b_{l}-b_{l+k}\right|
$$

It is easily shown that for any positive (nonzero) integer $n$,

$$
\begin{aligned}
\bar{d}(b, k) & =\frac{1}{n P} \sum_{l=0}^{n P-1}\left|b_{l}-b_{l+k}\right|=\frac{1}{P} \sum_{l=0}^{P-1}\left|b_{l}-b_{l+k}\right| \\
& =\frac{1}{P} \sum_{l=0}^{P-1}\left(b_{l}-b_{l+k}\right)^{2}=\frac{2}{P} \sum_{l=0}^{P-1} b_{l}^{2}-\frac{2}{P} \sum_{l=0}^{P-1} b_{l} b_{l+k}
\end{aligned}
$$

The auto-correlation function of a binary square wave, $s$, with $50 \%$ duty-cycle (mean 1/2) and period $P$ is a triangular wave of amplitude $1 / 2$ and period $P$, i.e.,

$$
\frac{1}{P} \sum_{l=0}^{P-1} s_{l} s_{l+k}=\frac{1}{2}(1-\operatorname{tri}(P, k))
$$


where $\operatorname{tri}(P, k)$ is given by

$$
\operatorname{tri}(P, k)=2\left|\frac{k}{P}-\left\lfloor\frac{k}{P}\right\rceil\right|
$$

and the function $\lfloor x\rceil$ rounds $x$ to the closest integer (ties can be resolved arbitrarily). This means that for binary periodic square waves with period $P$ we have from (9)

$$
\bar{d}(s, k)=\frac{2}{P} \sum_{l=0}^{P-1} s_{l}^{2}-\frac{2}{P} \sum_{l=0}^{P-1} s_{l} s_{l+k}=\frac{2}{P} \cdot \frac{P}{2}-2 \cdot \frac{1}{2}(1-\operatorname{tri}(P, k))=\operatorname{tri}(P, k)
$$

Theorem: The average distance spectrum $\bar{d}(k)$ defined by (4) of a binary reflected Gray code of order $m=\log _{2} M$ is

$$
\bar{d}(k)=\operatorname{tri}\left(2^{m}, k\right)+\sum_{i=2}^{m} \operatorname{tri}\left(2^{i}, k\right)
$$

Proof: In the list containing the codewords of the BRGC (cf. Table I), each of the $m$ columns, periodically repeated, represent binary square waves, the two leftmost with period $M$ and subsequent columns with periods $(M / 2, M / 4, \ldots, 4)$. Using (12) in (4) gives the desired result.

\section{REFERENCES}

[1] P. J. Lee, "Computation of the bit error rate of coherent $M$-ary PSK with Gray code bit mapping," IEEE Transactions on Communications, vol. COM-34, no. 5, pp. 488-491, May 1986.

[2] M. K. Simon, S. M. Hinedi, and W. C. Lindsey, Digital Communication Techniques, Prentice Hall, Englewood Cliffs, New Jersey, 1994.

[3] M. I. Irshid and I. S. Salous, "Bit error probability for coherent $M$-ary PSK systems," IEEE Transactions on Communications, vol. COM-39, no. 3, pp. 349-352, Mar. 1991.

[4] M. K. Simon and M. S. Alouini, Digital Communications over Fading Channels-A Unified Approach to Performance Analysis, John Wiley \& Sons, 2000.

[5] J. Lu, K. B. Letaief, J. C.-I. Chuang, and M. L. Liou, “ $M$-PSK and $M$-QAM BER computation using signal-space concepts," IEEE Transactions on Communications, vol. 47, no. 2, pp. 181-184, Feb. 1999.

[6] J. Lassing, E. Ström, T. Ottosson, and E. Agrell, “The exact symbol and bit error probabilities of coherent $M$-ary PSK," in Proc. IEEE International Symposium on Information Theory, Yokohama, Japan, June-July 2003.

[7] F. Gray, "Pulse code communications,” U. S. Patent No. 2632058, Mar. 1953.

[8] E. N. Gilbert, "Gray codes and paths on the n-cube," Bell System Technical Journal, vol. 37, pp. 815-826, May 1958.

[9] C. Savage, “A survey of combinatorial Gray codes,” SIAM Rev., vol. 39, no. 4, pp. 605-629, 1997. 


\section{TABLE I}

RECURSIVE CONSTRUCTION OF THE BRGCS OF ORDER $m=2,3$, AND 4 FROM THE BRGC OF ORDER $m=1$.

\begin{tabular}{|c|c|c|c|c|c|c|}
\hline$m=1$ & & $m=2$ & & $m=3$ & & $m=4$ \\
\hline 0 & $\Rightarrow$ & 00 & $\Rightarrow$ & 000 & $\Rightarrow$ & 0000 \\
\hline \multirow[t]{15}{*}{1} & & 01 & & 001 & & 0001 \\
\hline & & 11 & & 011 & & 0011 \\
\hline & & 10 & & 010 & & 0010 \\
\hline & & & & 110 & & 0110 \\
\hline & & & & 111 & & 0111 \\
\hline & & & & 101 & & 0101 \\
\hline & & & & 100 & & 0100 \\
\hline & & & & & & 1100 \\
\hline & & & & & & 1101 \\
\hline & & & & & & 1111 \\
\hline & & & & & & 1110 \\
\hline & & & & & & 1010 \\
\hline & & & & & & 1011 \\
\hline & & & & & & 1001 \\
\hline & & & & & & 1000 \\
\hline
\end{tabular}


TABLE II

AVERAGE DISTANCE SPECTRUM $\bar{d}(k)$ FOR THE BINARY REFLECTED GRAY (BRGG) AND THE CORRESPONDING VALUES USED IN [1] (LeE) FOR $M=2,4,8$, AND 16 . NOte THAT $\bar{d}(0)=0$ AND THAT ONLY VALUES FOR $k=1,2, \ldots, M / 2$ ARE GIVEN, $\operatorname{SINCE} \bar{d}(k)$ IS SYMMETRIC AROUND $k=M / 2$.

\begin{tabular}{|c|c|c|c|c|c|c|c|c|}
\hline \multirow[b]{2}{*}{$k$} & \multicolumn{2}{|c|}{$M=2$} & \multicolumn{2}{|c|}{$M=4$} & \multicolumn{2}{|c|}{$M=8$} & \multicolumn{2}{|c|}{$M=16$} \\
\hline & Lee & BRGC & Lee & BRGC & Lee & BRGC & Lee & BRGC \\
\hline 1 & 1 & 1 & 1 & 1 & 1 & 1 & 1 & 1 \\
\hline 2 & & & 2 & 2 & 2 & 2 & 2 & 2 \\
\hline 3 & & & & & 2 & 2 & 2 & 2 \\
\hline 4 & & & & & 2 & 2 & 2 & 2 \\
\hline 5 & & & & & & & 3 & $2.5(*)$ \\
\hline 6 & & & & & & & 3 & 3 \\
\hline 7 & & & & & & & 2 & $2.5(*)$ \\
\hline 8 & & & & & & & 2 & 2 \\
\hline
\end{tabular}




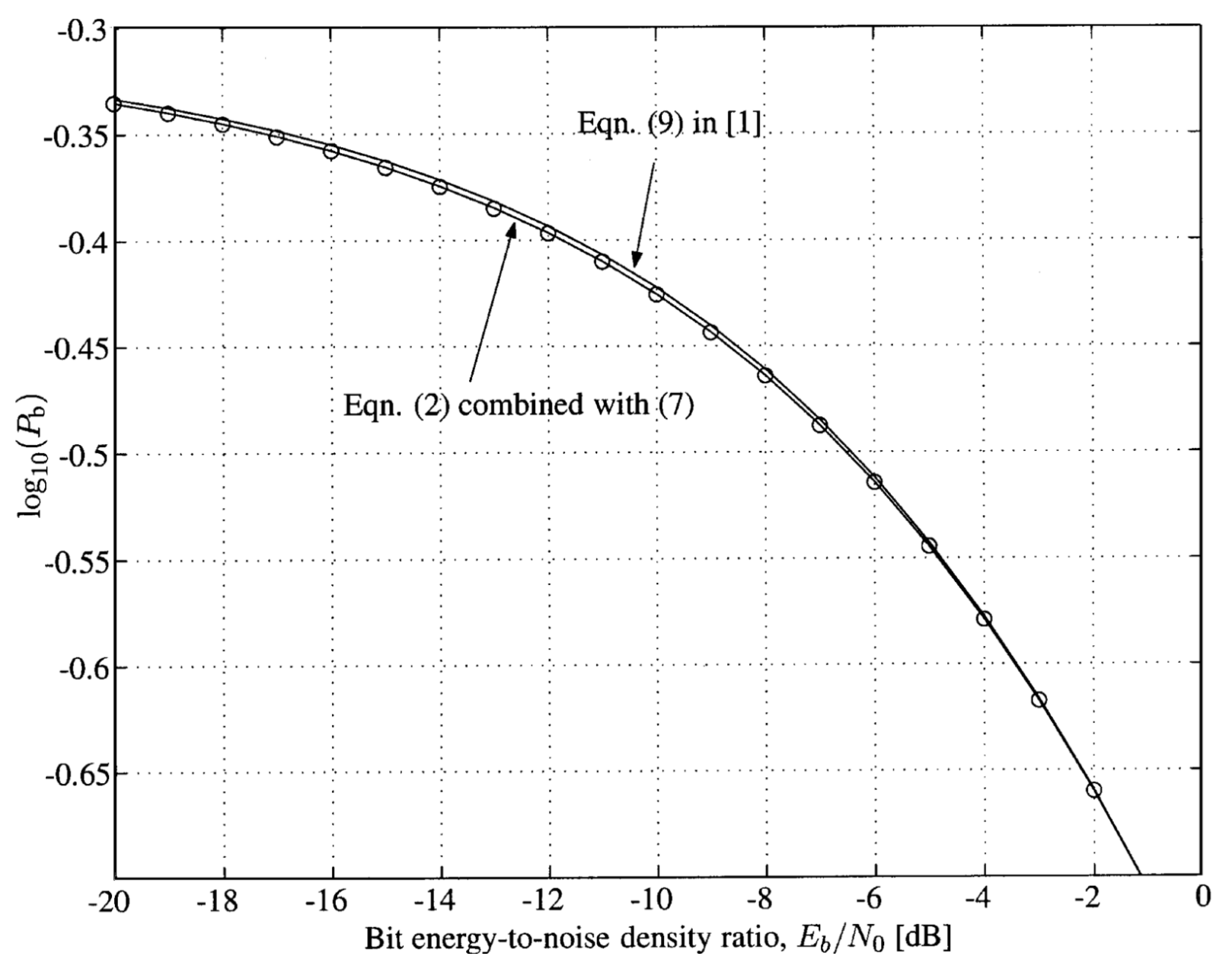

Fig. 1. Bit error probability of $M$-PSK for $M=16$. The exact expressions given by (2) together with (7) are plotted along with simulated values (०), compared with the previously proposed exact expression given in [1]. The difference is slight, but obvious. 